\title{
Dechlorination of halocarbons by microsomes and vesicular reconstituted cytochrome P-450 systems under reductive conditions
}

\author{
A G SALMON, ${ }^{1} \mathrm{~J}$ A NASH, ${ }^{2}$ C M WALKLIN,${ }^{3}$ AND R B FREEDMAN ${ }^{3}$ \\ From the TUC Centenary Institute of Occupational Health, ' London School of Hygiene and Tropical \\ Medicine, London WCIE 3HT, Central Toxicology Laboratory, ${ }^{2}$ Imperial Chemical Industries plc, \\ Macclesfield, Cheshire, and Biological Laboratory, ${ }^{3}$ University of Kent, Canterbury, UK
}

\begin{abstract}
A spectrophotometric assay of the reductive dechlorination of halocarbons was developed and used to determine the kinetic characteristics of dechlorination of a range of haloethanes catalysed by microsomes from rat and rabbit liver. Analysis of the typical reaction of hexachloroethane shows that the reaction is catalysed by cytochrome P-450 and results in the formation of olefinic products as well as less chlorinated alkanes: in other respects the reaction resembles that known to occur with carbon tetrachloride. The dechlorination of haloethanes catalysed by a vesicular reconstituted system of cytochrome P-450 enzymes from rabbit liver was also studied and found to be similar to that catalysed by microsomes: both reductase and a phenobarbital inducible form of cytochrome P-450 were essential. There is no substantial dependence of maximum dechlorination rate on compound structure, suggesting that the reduction of substrate is not the rate limiting step in the overall reaction. The main factor in determining the apparent binding constant to the enzyme is the partition coefficient into the lipid membrane, as assessed by calculated $\log P$ values.
\end{abstract}

The metabolism of halogenated hydrocarbons commonly involves dehalogenation, which in many cases is dependent on microsomal cytochrome P-450. Van Dyke and Wineman showed that the dechlorination of chloroalkanes was dependent on NADPH and inhibited by CO; activity was also inducible by phenobarbital but not by 3-methylcholanthrene.' Analysis of the reactivity of halocarbons as a function of their structure has been attempted on the assumption of a single mechanism. ${ }^{2}$ We have shown previously, however, the necessity of considering more than one mechanism in order to explain the behaviour of various compounds that have been studied in vitro. ${ }^{34}$ This is in agreement with the observations of products and mechanisms for those halocarbons that have been studied in detail: these data have been reviewed recently. ${ }^{5}$ It seems that most dechlorination reactions are oxidative in character, involving an initial attack by an oxygenated complex of cytochrome

Received 20 August 1984

Accepted 17 September 1984
$\mathrm{P}-450$ on a hydrogen atom, resulting in rearrangement of the product with the loss of a halogen. Nevertheless, at least one other important mechanism of dechlorination exists, which is observed when chemical structure or reaction conditions prevent the oxidative route. This mechanism, of which the metabolism of carbon tetrachloride is the best known example, involves reductive generation of a radical and direct release of a halide ion.

The objectives of this study were: firstly, to define a simple and reliable assay for the reductive reaction, based on the provision of a suitable anaerobic reaction medium. ${ }^{6}$ This needed to be sufficiently reproducible to allow proper kinetic analysis of the results. Secondly, to apply the assay and kinetic analysis to a range of haloethanes to investigate the structure/reactivity relationship for the reductive reaction. Thirdly, to test the role of cytochrome P-450 isoenzymes and NADPH:cyt P-450 reductase directly by studies using reconstituted systems. Previous studies have reported reconstitution of reductive dechlorination of carbon tetrachloride and halothane in non-vesicular systems comprising 
cytochrome P-450, reductase, lipid, and detergent. $^{78}$

To allow the use of the better defined and more easily prepared rabbit P-450 enzymes, the reaction was studied using both rabbit liver microsomes, with and without induction by phenobarbital and $\beta$-naphthoflavone, and also the rat liver microsomes that have been the subject of most previous work. These data were then compared with those obtained with purified cytochrome P-450 enzymes isolated from rabbit liver in a reconstituted system of vesicles containing reductase, lipid, and cytochrome. We have investigated the progress of this reaction under favourable conditions in vitro for a series of compounds that were expected to be susceptible to this process. Observed reactivities were correlated with structural features of the reacting species. We have also examined the metabolites formed in vitro from a typical compound, hexachloroethane. Further investigations performed with this compound were designed to examine the effects of the mixed function oxidase inhibitors carbon monoxide and SKF $525 \mathrm{~A}$ on the reaction.

\section{Methods}

\section{PREPARATION OF MICROSOMES}

Rat microsomes were prepared from the livers of normal (non-induced) male rats (Alderley Park strain, Wistar derived). The method of preparation has been described. ${ }^{9}$ Food was withdrawn 24 hours before death, and the washed microsomes were stored under argon at $-70^{\circ} \mathrm{C}$. Rabbit microsomes were prepared by the method of van der Hoeven and Coon ${ }^{10}$ from the livers of 3 month old male New Zealand White rabbits and stored at $-70^{\circ} \mathrm{C}$. For non-induced microsomes, the animals had access ad libitum to plain tap water for drinking, but for phenobarbital induction this was replaced by tap water to which sodium phenobarbital $(0 \cdot 1 \% \mathrm{w} / \mathrm{v})$ was added. Induction by $\beta$-naphthoflavone was achieved by intraperitoneal injections daily for three days at $20 \mathrm{mg} / \mathrm{kg}$ as a $1 \%(\mathrm{w} / \mathrm{v})$ suspension in corn oil.

All chemicals were of AnalaR grade or next highest available purity, and were obtained from $\mathrm{BDH}$, Poole, Dorset, unless noted to the contrary. (Tris buffer used was "Trizma" base enzyme grade material from Sigma (London) Chemical Co, Poole.) Ethoxyresorufin and resorufin were gifts from $\mathrm{Dr} T$ C Orton, ICI Pharmaceuticals Division. Benzphetamine $\mathrm{HCl}$ was a gift from Dr G G Gibson, University of Surrey. $\beta$-naphthoflavone was purchased from Aldrich Chemical Co, Gillingham.
SPECTROPHOTOMETRIC ASSAY OF

\section{DEHALOGENATION}

Stored microsomes were thawed gently and diluted with buffer (Tris $50 \mathrm{mM}$, EDTA $1 \mathrm{mM}$, pH 7.5) which had been deoxygenated by bubbling with nitrogen for one hour. The reaction mixture was prepared with the following components, also in deoxygenated buffer: microsomes $(1 \mathrm{mg} / \mathrm{ml})$, D-glucose $30 \mathrm{mM}$, Catalase, 2000 units $/ \mathrm{ml}$ (from bovine liver, purchased from Sigma), glucose oxidase 5 units/ml (from Aspergillus niger, type V, also purchased from Sigma), NADPH $500 \mu \mathrm{mol}$. Samples of the resulting preparation were placed in sealed cuvettes under nitrogen. All transfers were carried out under nitrogen with flushed syringes, containers being sealed with silicone rubber septa or stoppers. The cuvettes were placed in a Beckman Acta MVI or a Perkin Elmer 356 spectrophotometer fitted in both cases with a scattering sample attachment. Absorbance at $340 \mathrm{~nm}$ was monitored, a "baseline" rate of change without substrate being established first. The substrate was added to the sample cuvette as a solution in $1 \mu \mathrm{l}$ spectroscopic grade ethanol. The change in absorbance at $340 \mathrm{~nm}$ was observed as a function of time, and the initial rate determined. Spectrophotometric assays were performed in a similar manner with a reconstituted cytochrome P-450 system (see below). The kinetic parameters $K_{m}$ and $V_{\max }$ were determined by a computer program"1 which uses the direct linear plot $^{12}$ and also fits regression lines to the three standard linear transformations of the Michaelis-Menten Equation. The halocarbon susbstrates were obtained from Aldrich Chemical Co, Gillingham.

To study the effect of SKF525A on the reaction of hexachloroethane, the compound (a gift from Smith, Kline and French, Welwyn) was added in buffer. In the similar experiment with carbon monoxide, the reaction mixture was saturated with the gas by bubbling for two minutes.

\section{IDENTIFICATION OF REACTION PRODUCTS}

For aerobic incubations, $25 \mathrm{ml}$ flasks sealed with silicone septa were prepared, containing the following reagents in a total volume of $10 \mathrm{ml}: \mathrm{KCl}(75$ mmol), D-glucose-6-phosphate ( $5 \mathrm{mmol}$ ), NADP+ (250 $\mu \mathrm{mol})$, nicotinamide $(4.5 \mathrm{mmol})$, glucose-6phosphate dehydrogenase $(0.25$ units $/ \mathrm{ml})$, in Tris buffer (as above). For anaerobic incubations, an oxygen scavenging system was incorporated in the reaction system. This consisted of $\mathrm{D}$-glucose, catalase, and glucose oxidase, as used in the spectrophotometric assay. All solutions used in anaerobic incubations were deoxygenated before use, and the headspace of the flask was purged with nitrogen. 
${ }^{14} \mathrm{C}$-hexachloroethane (final concentration $2 \mu \mathrm{M}$, $41.44 \mu \mathrm{Ci}$ radioactivity) was added in $20 \mu \mathrm{l}$ ethanol to the liquid phase and the flasks incubated at $37^{\circ} \mathrm{C}$ in a shaking water bath. Flasks were sampled immediately after injection of substrate and after 145 minutes. Control incubations with boiled microsomes were run concurrently. The headspace was sampled $(1 \mathrm{ml})$ with a gas-tight syringe, and analysed by gas liquid chromatography. Two columns were used as necessary to separate and identify the labelled metabolite peaks. These were a $1.5 \mathrm{~m} \times$ $2 \mathrm{~mm}$ id glass column packed with $5.5 \%$ OV101 on Supelcoport (100-200 mesh), maintained at $80^{\circ} \mathrm{C}$, and a $2 \mathrm{~m} \times 2 \mathrm{~mm}$ id glass column packed with 60/80 Carbopack B/1\% SP1000 (Supelco Inc), maintained at $175^{\circ} \mathrm{C}$. Gas chromatography materials were obtained from Phase Separations Ltd (Clwyd). A Pye 104 gas chromatograph with flame ionisation detector and a radiochemical detector (ESI Nuclear) was used. Metabolite peaks were identified by comparison of retention times and by cochromatography with authentic standards. The gas-tight syringe was purged with nitrogen and checked for carry over between samples.

The radiolabelled hexachloroethane $(21.5 \mathrm{mCi} /$ mmol) used was obtained from Physics and Radioisotopes Services, ICI Petrochemicals and Plastics Division, Billingham. This material was purified by gas liquid chromatography and had a radiochemical purity of better than $98 \%$. No impurity peaks were detected on analysis of the material by gas liquid chromatography, either with the flame ionisation or radioactivity detectors.

\section{RECONSTITUTED SYSTEMS}

Purified preparations of rabbit cytochromes P-450 (LM2 and LM4) were derived by a procedure involving polyethylene glycol fractionation of cholate solubilised, pyrophosphate washed microsomes, followed by ion-exchange chromatography on DEAE-Sephacel, adsorption chromatography on spheroidal hydroxyapatite and calcium phosphate (all in the presence of Renex 690), treatment with Amberlite XAD-2 to remove detergent, and chromatography on Octyl-Sepharose 4B. The method is modified from the procedures of van der Hoeven and Coon ${ }^{10}$ and Wolf et al ${ }^{7}$ : full details are given by Walklin. ${ }^{13}$ NADPH:cytochrome $\mathrm{P}-450$ reductase was purified by the method of French and Coon. ${ }^{14}$ Reconstruction of these proteins in vesicles with dilaurylglycerylphosphatidylcholine (Fluka AG, Buchs, Switzerland, synthetic, $98 \%$ pure) was by the cholate gel filtration method. ${ }^{15}$ The vesicles eluted at the void volume from columns of Sephadex G50 (Medium) and electron microscopy showed them to be roughly spherical, having diameters in the range $150-200 \mathrm{~nm}$. SDS-polyacrylamide gel electrophoresis analysis showed that close to $100 \%$ of the added enzymes had been incorporated into the vesicles.

\section{OTHER ASSAYS}

Mixed function oxidase activities towards standard substrates were assayed: ethoxyresorufin O-deethylation, ${ }^{16}$ and benzphetamine $\mathrm{N}$ demethylation. ${ }^{17}$ Protein concentrations were measured by the method of Lowry et al ${ }^{18}$ modified for use with membrane proteins, ${ }^{14}$ using crystalline bovine serum albumen as a standard. Cytochrome P-450 content was assayed spectrophotometically ${ }^{20}$ : the specific cytochrome P-450 contents of the purified preparations used in this work were: LM2 12.5 $\mathrm{nmol} / \mathrm{mg}, \mathrm{LM} 411.7 \mathrm{nmol} / \mathrm{mg}$. NADPH:cytochrome $\mathrm{P}-450$ reductase was assayed using cytochrome $\mathrm{c}$ as oxidant $^{21}$; the stock preparation used here had an activity of 52.5 units $/ \mathrm{mg}$. (One unit catalyses the reduction of $1.0 \mu \mathrm{mol}$ cytochrome c per minute: $0.3 \mathrm{M}$ phosphate buffer $\mathrm{pH} 7 \cdot 7,30^{\circ} \mathrm{C}$.)

\section{Results and discussion}

The anaerobic assay system allowed the reliable determination of rates of cytochrome P-450 dependent reductive dechlorination reactions catalysed by liver microsomes. In the absence of added substrate the rate of NADPH oxidation was negligible, and on addition of substrate it proceeded linearly for at least two minutes. Using rat liver microsomes with hexachloroethane as substrate, $98 \%$ was inhibited by saturation of the assay mixture with $\mathrm{CO}$ and $37 \%$ inhibited by $100 \mu \mathrm{mol}$ SKF $525 \mathrm{~A}$. When phenobarbital induced rabbit liver microsomes were used the CO inhibition approached $100 \%$.

The incubation of microsomes $(1.377 \mathrm{mg} / \mathrm{ml}, 1.4$ nmoles P450 per $\mathrm{mg}$ protein) and an NADPH generating system with hexachloroethane followed by gas chromatographic analysis of the headspace showed the formation of tetrachloroethane as the major product, under conditions of normal oxygen partial pressure and also anaerobically (fig 1). The rate under anaerobic conditions seemed to be greater than in the presence of oxygen: although exact quantitation of total product is not possible using the headspace method, the amount of product formed under aerobic conditions appeared to be between $10 \%$ and $20 \%$ of that formed under anaerobic conditions. A second product, trichloroethylene, was observed under aerobic conditions in small quantities, but was produced in larger amounts in the anaerobic incubation. The other product observed was pentachloroethane. Under aerobic conditions the peak corresponding to this compound was no 


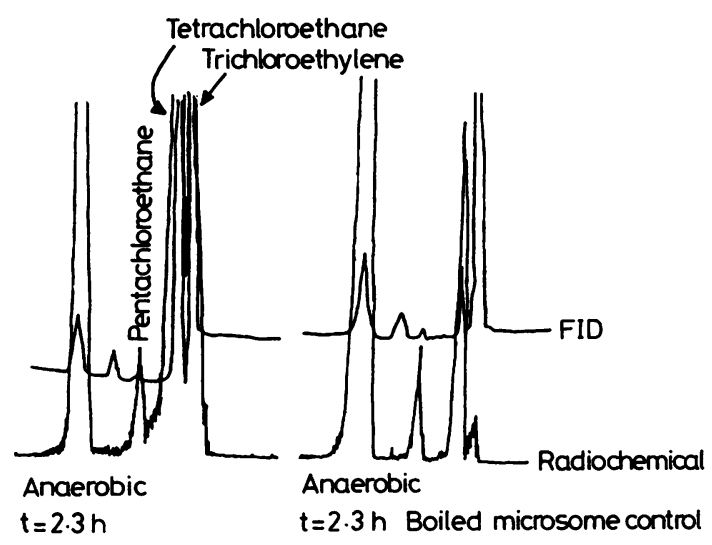

Fig 1 Gas liquid chromatography of headspace products of a dechlorination reaction catalysed by rat liver microsomes under anaerobic conditions. Conditions of incubation were as described in text, and chromatography was on a column consisting of $5 \%$ OV101 on Supelcoport.

larger than that seen in the boiled microsome control incubation, but a significant increase in the amount of this material in the headspace $(150 \%)$ was seen under anaerobic conditions. The presence of significant amounts of material corresponding to products in the boiled microsome control incubations was noted, but this observation was not repeated in control incubations lacking only substrate or microsomes. It was therefore concluded that in addition to the enzyme catalysed reaction, a small catalytic effect is shown by a heat-stable or heat generated component of the boiled microsomes, possibly $\mathrm{Fe}^{+++}$. Substantial amounts of radioactivity were shown to remain in the aqueous phase after the complete incubations, some but not all of which was

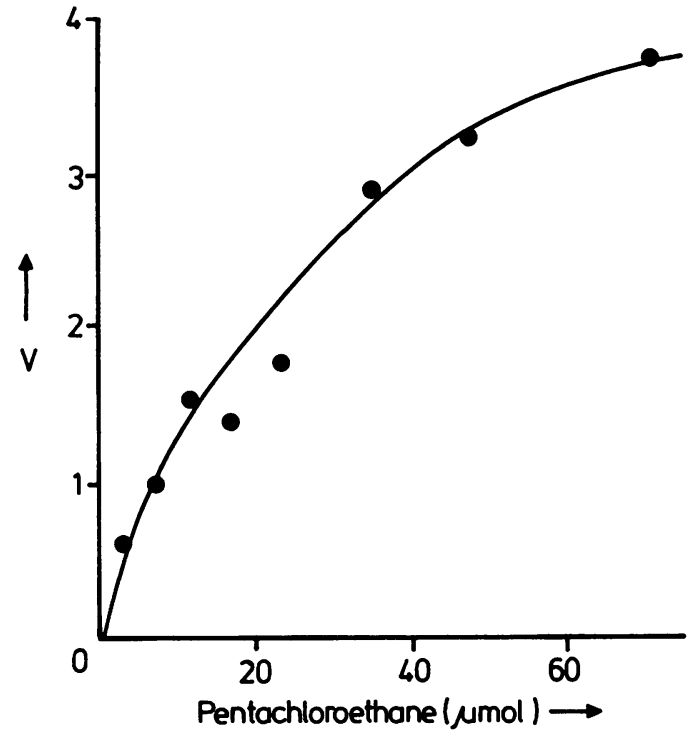

Fig 2 Reductive dechlorination of pentachloroethane catalysed by rat liver microsomes. Initial velocities observed in spectrophotometric assay described above are displayed as a function of substrate concentration.

unchanged hexachloroethane $-{ }^{14} \mathrm{C}$.

The inhibitor effects and the nature of the products indicate that the reaction being assayed spectrophotometrically is a cytochrome $\mathrm{P}-450$ dependent reductive dechlorination. It has been concluded on the basis of similar observations ${ }^{22}$ that these products resulted from a two electron reduction of the substrate. The reaction resembles the reductive dechlorination of carbon tetrachloride except that the intermediate radical product is readily suscept-

Table 1 Kinetic parameters for reductive dechlorination of halocarbons by control rat liver microsomes

\begin{tabular}{|c|c|c|c|}
\hline Compound & $K_{m}(\mu M)$ & 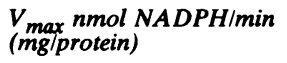 & $\begin{array}{l}V_{\text {max }} \text { nmol NADPH/min } \\
\text { nmole } P-450\end{array}$ \\
\hline $\begin{array}{l}\text { Carbon tetrachloride } \\
\text { Hexachloroethane } \\
\text { Pentachloroethane } \\
1,1,1,2 \text {-tetrachloroethane } \\
1,1,2,2 \text {-tetrachloroethane } \\
\text { 1,1,1-trichloroethane } \\
\text { 1,1-dichloroethane } \\
\text { 1,2-dichloroethane } \\
\text { 1,1,1-trichloro-2,2,2-trifluorethane } \\
1,1,1,2 \text {-tetrachloro-2,2-difluoroethane }\end{array}$ & $\begin{array}{l}9 \cdot 6 \\
6 \cdot 0 \\
25 \cdot 1^{*} \\
20 \cdot 4^{*} \\
\text { (a) } \\
\text { (b) } \\
\text { (a) } \\
\text { (a) } \\
11 \cdot 7 \\
25 \cdot 9\end{array}$ & $\begin{array}{l}3 \cdot 35 \\
3 \cdot 55^{*} \\
4 \cdot 35^{*} \\
3 \cdot 41 \\
\text { (a) } \\
\text { (b) } \\
\text { (a) } \\
\text { (a) } \\
1 \cdot 78 \\
4 \cdot 49\end{array}$ & $\begin{array}{l}2 \cdot 15 \\
2 \cdot 41 \\
3 \cdot 35 \\
2 \cdot 62 \\
\text { (a) } \\
\text { (b) } \\
\text { (a) } \\
\text { (a) } \\
1 \cdot 37 \\
3 \cdot 45\end{array}$ \\
\hline
\end{tabular}

Kinetic parameters were derived from Direct Linear, Eadie-Hofstee, and Lineweaver-Burk plots of the data. The Lineweaver-Burk plot is known to be most prone to error when there is experimental scatter in data. Parameters quoted are those obtained from a regression line of best fit to data plotted as an Eadie-Hofstee plot; except in a few cases $\left({ }^{*}\right)$ the values obtained from Direct Linear plot are within $10 \%$ of values quoted.

(a) Rates of NADPH oxidation observed were too low to allow determination of kinetic parameters.

(b) NADPH oxidation was observed at a low rate, but protein precipitation interfered with measurements at substrate levels high enough to allow determination of kinetic parameters. 
Table 2 Water: octanol partition data

\begin{tabular}{ll}
\hline Compound & Log $P$ \\
\hline Carbon tetrachloride & $2 \cdot 96$ \\
Hexachloroethane & $4 \cdot 62$ \\
Pentachloroethane & $3 \cdot 69$ \\
1,1,1,2-tetrachloroethane & $3 \cdot 05$ \\
1,1,2,2-tetrachloroethane & $2 \cdot 72$ \\
1,1,1-trichloroethane & $2 \cdot 50$ \\
1,1-dichloroethane & $1 \cdot 80$ \\
1,2-dichloroethane & $1 \cdot 48$ \\
1,1,1-trichloro-2,2,2-trifluorethane & $3 \cdot 26$ \\
\hline
\end{tabular}

Log $P$ is a calculated octanol:water partition coefficient, derived by Mr P J Taylor, ICI Pharmaceuticals Division, using the method of Leo et al. . $^{23}$

ible to further reduction to the olefin in the case of the two-carbon substrates, whereas for carbon tetrachloride this material is not so readily subject to further metabolism and tends to accumulate. For both the haloethanes and carbon tetrachloride this reductive reaction, although more readily detected under anaerobic conditions, continues at a substantial rate even at the atmospheric partial pressure of oxygen, which is higher than that to be expected in some tissues-for example, those parts of the liver acinus closer to the central vein. It is therefore supposed that this type of reaction is of physiological and pathological importance.

The rate of reductive dechlorination by control rat liver microsomes was assayed spectrophotometrically with a large number of haloethane substrates, at concentrations between 2 and $500 \mu \mathrm{mol}$. In general the data are in good agreement with the Michaelis-Menten equation (see fig 2). These data were analysed to provide values of $\mathrm{K}_{\mathrm{m}}$ and $\mathrm{V}_{\max }$,

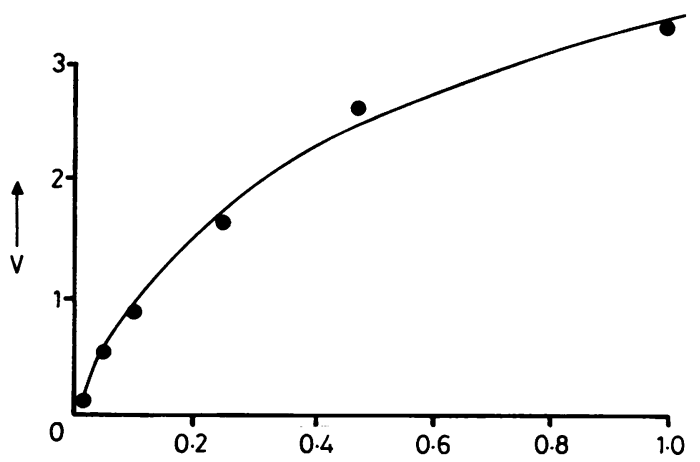

(1,1,1-Trichloroethane) $\mathrm{mmol} \longrightarrow$

Fig 3 Reductive dechlorination of 1,1,1-trichloroethane catalysed by purified rabbit liver cytochrome P-450 (LM2). Conditions for the spectrophotometric assay were as described for vesicular reconstituted systems, initial velocities being presented as a function of substrate concentration.
Table 3 Kinetic parameters for reductive dechlorination of chloroethanes by a vesicular reconstituted system

\begin{tabular}{lcl}
\hline Compound & $K_{m}(\mu M)$ & $\begin{array}{l}V_{\text {max }} \text { nmol NADPH/min } \\
\text { nmols0 }\end{array}$ \\
\hline Hexachloroethane & 50 & 2.39 \\
Pentachloroethane & $104^{*}$ & 1.48 \\
1,1,2,2-tetrachloroethane & 119 & 1.66 \\
1,1,1-trichloroethane & 360 & 4.33 \\
1,1-dichloroethane & 802 & 1.27 \\
\hline
\end{tabular}

See legend to table 1 .

which are reported for a number of substrates in table 1. Similar observations were made for rabbit liver microsomes, although a rather higher absolute rate was observed, especially after phenobarbital induction.

With both rat and rabbit microsomes the major finding is that there is limited variation in $\mathrm{V}_{\max }$, but considerably greater variation in $\mathrm{K}_{\mathrm{m}}$. The lack of dependence of $V_{\max }$ on structural features of the haloethanes suggests that in this reductive dechlorination the substrate reaction is not rate limiting. The interpretation of $K_{m}$ values for the interaction of non-polar substrates with membrane bound enzymes is complicated by the partition of the substrate between the aqueous and the membrane phases; the values obtained neglect this partition and are therefore not true measures of the dissociation constant describing the equilibrium between free substrate and enzyme/substrate complex. Hence it is dangerous to attempt structure/activity interpretations of these crude $K_{m}$ values. In general it is found that the $K_{m}$ values decrease with increasing extent of chlorination of the substrates; this may reflect the increasingly non-polar nature of the more highly substituted substrates, and hence their greater partition into the membrane phase and their concentration in the environment of the enzyme. The polarities of the various compounds are illustrated in terms of their water-octanol partition coefficients (measured or derived) given in table 2 . If it is assumed that substrates partition between bulk aqueous phase and membrane as determined by these constants and that substrate in the membrane phase then equilibrates with the substrate binding site on the cytochrome, the "true" $K_{m}$ describing this binding equilibrium is given by:

$$
K_{m}=\frac{K_{m}{ }^{a p p} \cdot P \lambda}{1+P \lambda}
$$

where $K_{m}{ }^{\text {app }}$ is the value derived directly from kinetic studies, $P$ is the partition conefficient and $\lambda$ is the volume fraction occupied by the membrane phase. ${ }^{24}{ }^{25}$ Using this approach the true $\mathrm{K}_{\mathrm{m}}$ values 
for the chloroethanes with rat liver microsomes are in the region of $16 \mathrm{mmol}$.

Involvement of particular cytochrome P-450 species in the reductive dechlorination is suggested by studies with liver microsomes from animals pretreated with inducers. Microsomes from $\beta$-naphthoflavone induced rabbits did not catalyse the reaction at a measurable rate with any of the six haloethanes tested, and microsomes from control rabbits gave a measurable rate of reaction at high concentrations of hexachloroehtane only. On the other hand, those from phenobarbital induced rabbits catalysed the reaction with several compounds. $\mathrm{K}_{\mathrm{m}}$ values for these microsomes ranged from $<5 \mu \mathrm{mol}$ for hexachloroethane to about $80 \mu \mathrm{mol}$ for 1,1,2,2-tetrachloroethane.

These findings suggest that the reductive dechlorination reaction is preferentially catalysed by a cytochrome P-450 isoenzyme that is induced by phenobarbital, possibly the major phenobarbital inducible form. This hypothesis was tested by the isolation from rabbit liver microsomes of the major phenobarbital induced isoenzyme (LM2), and the isolation of the major aromatic hydrocarbon inducible form (LM4) for comparison. These proteins were isolated rapidly and in good yield by a new combination of chromatographic techniques described fully elsewhere. ${ }^{12}$ The purified cytochrome P-450s were reconstituted with and without rabbit liver NADPH:cytochrome $\mathrm{P}-450$ reductase, in defined lipid bilayer vesicles of dilauryl phosphatidyl choline. Vesicles containing LM2 and reductase were active in the NADPH dependent demethylation of benzphetamine, but not the NADPH dependent de-ethylation of ethoxyresorufin, whereas the opposite was true of vesicles containing LM4 and reductase. No mixed function oxidase activities were observed with vesicles containing cytochrome P-450 alone or reductase alone.

Vesicles containing cytochrome P-450 LM2 and reductase were active in the NADPH dependent reductive dechlorination or haloethanes. Figure 3 shows kinetic data obtained in the reaction with 1,1,1-trichloroethane: kinetic data for a range of substrates are shown in table 3 . Interestingly, vesicles containing LM2 and reductase catalyse reactions of a slightly wider range of haloethane substrates at measurable rates than do phenobarbital induced microsomes (table $3 \mathrm{cf}$ table 1). Comparison of the kinetic data with various substrates shows that again there is little variation in $V_{\max }$ (3-fold), and considerably greater variation in the $K_{m}$ (16fold). As is the case with microsomes, $K_{m}$ values decreased with increasing extent of halogen substitution. Adopting the approach described above to calculate "true" $K_{m}$ values yields for all substrates values in the region of $80 \mathrm{mM}( \pm 50 \%)$. Figure 4 shows the effect of $\log \mathrm{P}$ in compensating for the variation in apparent $K_{m}$, assuming the substrates bind from the membrane phase. Thus in this system it appears that neither $V_{\max }$ nor the "true" $K_{m}$ is strongly dependent on the nature of the substrate and that the rate determining step may be independent of substrate. No activity was observed with vesicles containing LM2 alone or reductase alone, nor with vesicles containing LM4 and reductase. The reaction is therefore catalysed only by certain cytochrome P-450 isoenzymes, and is entirely dependent on a flow of electrons from NADPH via NADPH:cytochrome $\mathrm{P}-450$ reductase. The possibility that NADPH, cytochrome $b_{s}$ and NADPH: cytochrome $b_{5}$ reductase could also participate as electron donors to cytochrome P-450 was not investigated.

The data presented here are the first direct evidence that the reductive dechlorination pathway shown for a wide range of haloethanes is a specific property of certain cytochrome P-450 isoenzymes.

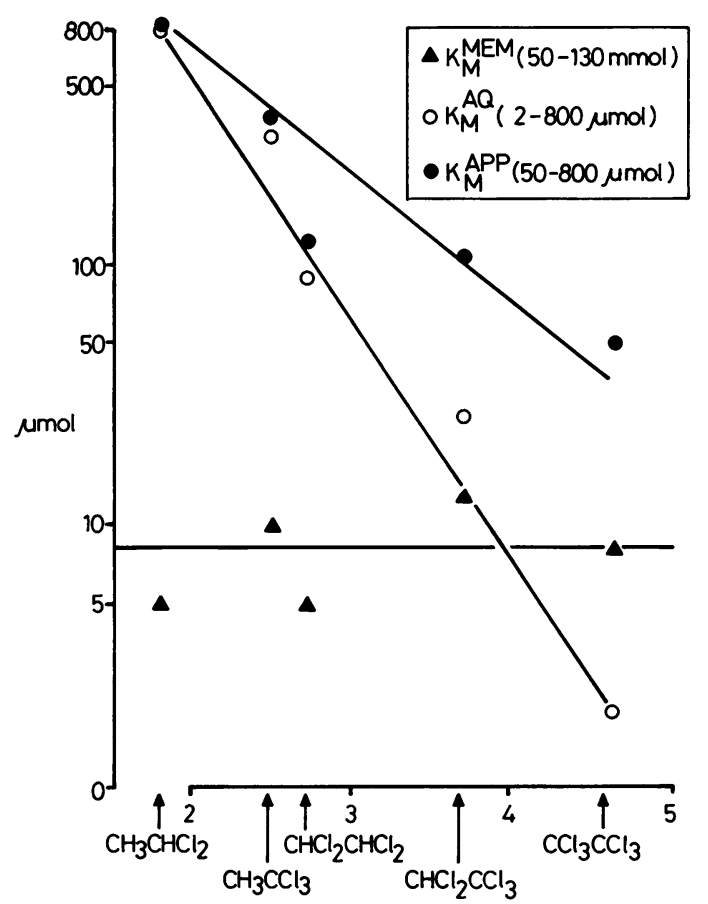

Fig 4 Variation of $K_{m}$ with $\log P$ when calculated on basis of total substrate (O), substrate present in the membrane phase ( $\mathbf{\Delta})$, and substrate present in the aqueous phase $(O)$, partitioning of substrate being calculated as described in discussion. 
The Science and Engineering Research Council and Imperial Chemical Industries plc (Central Toxicology Laboratory: director, Dr I F H Purchase) have supported this work by providing a studentship under the CASE scheme for $\mathrm{C} M$ Walklin. The investigations were carried out partly at the Biological Laboratory, University of Kent, and partly at ICI Central Toxicology Laboratory. Our thanks are due to Dr C Elcombe (ICI) for making various experimental facilities available and for helpful discussions. We also thank Dr P R McIntosh and Dr C R Woy for advice on methods of purification of cytochrome P-450, and Mr P J Taylor (ICI Pharmaceuticals Division) who calculated the $\log \mathrm{P}$ values for us.

\section{References}

I Van Dyke RA, Wineman CG. Enzymatic dechlorination: dechlorination of chloroethanes and chloropropanes in vitro. Biochem Pharmacol 1971;20:463-70.

${ }^{2}$ Loew G, Trudell J, Motulsky H. Quantum chemical studies of the metabolism of a series of chlorinated ethane anaesthetics. Mol Pharmacol 1973;9:152-62.

${ }^{3}$ Salmon AG, Mackrodt WC, Jones RB, Basu SK, Ashby J. An investigation of the dechlorination of a series of haloalkanes by rat liver microsomes and its possible role in the expression of toxic phenomena. Toxicol Appl Pharmacol 1978;45:327. (Abstract.)

4 Salmon AG, Jones RB, Mackrodt WC. Microsomal dechlorination of haloethanes-structure/reactivity relationships. Xenobiotica 1981;11:723-34.

s Anders MW. Aliphatic halogenated hydrocarbons. In: Jakoby WB, Bend JR, Caldwell J, eds. Metabolic basis of detoxification: metabolism of functional groups. New York: Academic Press, 1982.

- Wolf CR, King LJ, Parke DV. Anaerobic dechlorination of trichlorofluoromethane by liver microsomal preparations in vitro. Biochem Soc Trans 1978;3:175-7.

' Wolf CR, Slaughter SR, Marciniszyn JP, Philpott RM. Purification and structural comparison of pulmonary and hepatic cytochrome P-450 from rabbits. Biochim Biophys Acta 1980;624:409-19.

${ }^{8}$ Fujii K, Miki N, Sugiyama T, Morio M, Yamano T, Miyake Y. Anaerobic dechlorination of halothane by reconstituted liver microsomal cytochrome P-450. Biochem Biophys Res Comm 1981; 102:507-12.

'Salmon AG. Cytochrome P-450 and the metabolism of vinyl chloride. Cancer Lett 1976;2:109-14.

${ }^{10}$ Van der Hoeven TA, Coon MJ. Preparation and properties of partially purified cytochrome $\mathrm{P}-450$ and NADPH-cytochrome P450 reductase from rabbit liver microsomes. J Biol Chem 1974; 249:6302-10.

"Pettit N. Investigations of the characteristics and stability of accumulated soil enzymes. Canterbury: University of Kent, 1977. (PhD thesis.)

12 Eisenthal R, Cornish-Bowden A. The direct linear plot-a new graphical procedure for estimating enzyme kinetic parameters. Biochem J 1974;139:715-20.

${ }_{13}$ Walklin CM. Organisational and enzymological properties of the microsomal mono oxygenase system. Canterbury: University of Kent, 1984. (PhD thesis.)

${ }^{14}$ French JS, Coon MJ. Properties of NADPH-cytochrome P-450 reductase purified from rabbit liver microsomes. Arch Biochem Biophys 1979;195:565-72.

is Ingelman-Sundberg M, Glaumann H. Incorporation of purified components of the rabbit liver microsomal hydroxylase system into phospholipid vesicles. Biochim Biophys Acta 1980;599:417-35.

${ }^{16}$ Burke MD, Mayer RT. Ethoxyresorufin: direct fluorimetric assay of a microsomal dealkylation which is preferentially inducible by 3-methylcholanthrene. Drug Metab Dispos 1974;2:583-8.

${ }^{17}$ Haugen DA, van der Hoeven TA, Coon MJ. Purified liver microsomal cytochrome P-450: separation and characterisation of multiple forms. J Biol Chem 1975;250:3567-70.

${ }^{18}$ Lowry OH, Rosebrough HJ, Farr AC, Randall RJ. Protein measurement with the Folin phenol reagent. J Biol Chem 1951;193:265-73.

19 Sandermann H, Strominger JL. Purification and properties of $\mathrm{C}_{35}$ isoprenoid alcohol phosphokinase from staphylococcus aureus. J Biol Chem 1972;247:5123-31.

${ }^{20}$ Omura T, Sato $R$. The carbon monoxide-binding pigment of liver microsomes. ii. solubilisation, purification and properties. $J$ Biol Chem 1964;239:2379-85.

${ }^{21}$ Phillips AH, Langdon RG. Hepatic triphosphopyridine nucleotide-cytochrome c reductase: isolation, characterisation and kinetic studies. J Biol Chem 1962;237:2652-60.

${ }^{22}$ Nastainczyk W, Ahr HJ, Ulrich V. The reductive dechlorination of halogenated alkanes by microsomal cytochrome P-450. Biochem Pharmacol 1982;31:391-6.

${ }^{23}$ Leo A, Jow PYC, Silipo C, Hansch C. Calculation of the hydrophobic constant ( $\log P)$ from pi and f constants. J Med Chem 1975;18:865-8.

${ }^{24}$ Freedman RB. In: Finean JB, Michell RH, eds. Structural aspects of biological membranes. New comprehensive biochemistry. Vol 1. Amsterdam: Elsevier-North Holland, 1981:161.

${ }^{25}$ Parry G, Palmer DN, Williams DJ. Ligand partitioning into membranes: its significance in determining $K_{m}$ and $K_{s}$ values for cytochrome P-450 and other membrane-bound receptors and enzymes. FEBS Lett 1976;67:123-9. 